\title{
Limited Effect of Twin Boundaries on Radiation Damage
}

\author{
Jin Gao ${ }^{1} \cdot$ Zhi-Jun Liu ${ }^{1} \cdot$ Fa-Rong Wan ${ }^{1}$
}

Received: 16 October 2015/Revised: 8 November 2015/Published online: 4 January 2016

(C) The Chinese Society for Metals and Springer-Verlag Berlin Heidelberg 2015

\begin{abstract}
The different influences of the general grain boundary and the twin boundary on the cavity formation were investigated in copper irradiated by helium at room temperature. Large number of the cavities with sizes smaller than $5 \mathrm{~nm}$ formed after irradiation, which were almost homogenously distributed inside the grains. However, the distribution of the cavities varied near the general grain boundary and the twin boundary. Cavities-depleted zone was readily observed near the general grain boundaries, which was rarely observed near the twin boundaries. Meanwhile, decoration of dense cavities within the grain boundary plane was observed. The results suggested the limited influence of the twin boundaries as the point defect sinks compared with the general grain boundaries.
\end{abstract}

\section{KEY WORDS: Transmission electron microscopy; Cavities; Cavities-depleted zone; General grain boundary; Twin boundary}

\section{Introduction}

It has long been recognized that the materials in high quality are key to the success of future fusion reactors [1] because of the severe working environment. Point defects, i.e., vacancies and interstitials, form during the displacement cascade. These point defects can cluster to form other types of defects, altering the mechanical property of the irradiated materials. It is speculated that the grain boundaries and the interfaces may serve as effective sinks for the point defects so they can remove the point defects increasing the radiation tolerance [2]. Similarly, nanocrystalline metals showed improved radiation resistance compared with their coarse-grain counterparts [3-7].

Available online at http://link.springer.com/journal/40195

Fa-Rong Wan

wanfr@mater.ustb.edu.cn

1 Department of Materials Physics and Chemistry, School of Materials Science and Engineering, University of Science and Technology Beijing, Beijing 100083, China
Bai et al. [8] revealed that interstitials re-emitted from the grain boundaries annihilated the less mobile vacancies in the bulk. It also showed that the interstitial-loaded grain boundaries could reduce the vacancy diffusion barrier so the vacancies could migrate to the grain boundaries more easily. Previous studies have shown the effect of the grain boundary on the radiation damage [9-15]. Thorsen et al. [9] reported their studies on helium bubble formation at the grain boundaries in helium-implanted copper at elevated temperature. Larger helium bubbles precipitated at the grain boundaries. Sakaguchi et al. [10] and Tomozawa et al. [11] studied the radiation-induced segregation in stainless steel, where no solute segregation was observed at the twin boundaries. Defect-depleted zone was also observed in neutron irradiated materials [13, 15]. However, fewer studies focused on the issue of the twin boundaries. Recently, some researchers even suggested that introducing high density of the twin boundaries to materials can be an effective way to improve the irradiation tolerance. From our perspective, the twin boundaries may have limited impact on the healing of radiation damage. 
In the present study, the different influences of two types of the grain boundaries, i.e., general grain boundaries and the twin boundaries, on the cavity formation were studied by transmission electron microscopy (TEM) in copper after helium irradiation at room temperature. The limited effect of the twin boundary as defect sinks was demonstrated.

\section{Experimental}

Pure thin film of $\mathrm{Cu}$ was mechanically polished to a thickness of $0.1 \mathrm{~mm}$, and then disks of $3 \mathrm{~mm}$ in diameter were punched out. The TEM specimens were prepared by twin-jet polishing in Tenupol-5 with a solution of $25 \%$ nitric acid and $75 \%$ methanol and washed in ethanol.

Helium ions of $30 \mathrm{keV}$ were implanted to TEM specimens at room temperature to a fluence of $1 \times 10^{17}$ ions/ $\mathrm{cm}^{2}$. Irradiation damage was calculated using the Stopping and Range of Ions in Matter (SRIM-2008) software as shown in Fig. 1. In the typical TEM observation region $(100-150 \mathrm{~nm})$, the average irradiation dose was $\sim 5.2 \mathrm{dpa}$ and the average helium concentration was $\sim 7.0$ at $\%$. The post-radiation analysis was performed by TEM (FEI Tecnai F20).

\section{Results}

\subsection{Irradiation Damage in Helium-irradiated $\mathrm{Cu}$}

The main characteristic of the radiation damage observed in this study was the formation of high density of small cavities inside the grain, as shown in Fig. 2a, b, recorded with over-focus and under-focus condition respectively. The areal density of the cavities was measured to be in the order of $10^{16} \mathrm{~m}^{-2}$. The small cavities almost uniformly

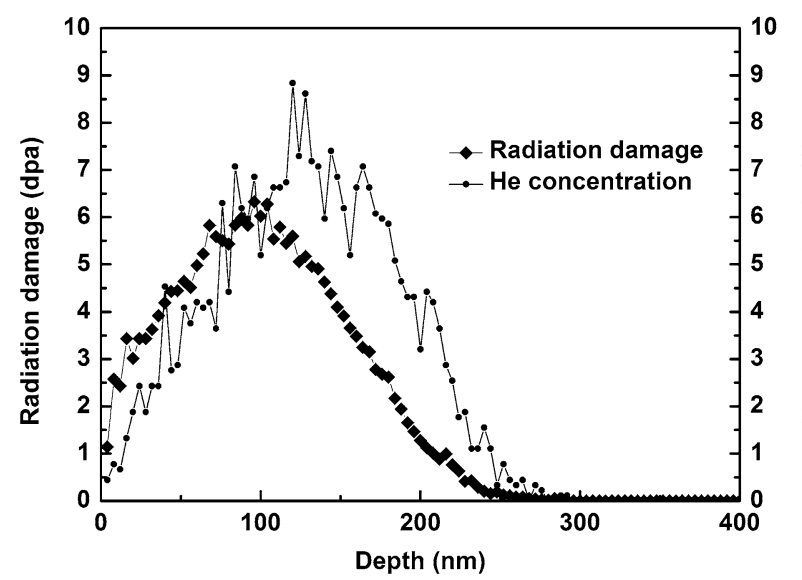

Fig. 1 SRIM calculation of irradiation damage and He concentration profile in $\mathrm{Cu}$ under $30 \mathrm{keV}$ helium irradiation distributed in the interior of the grain with comparable sizes. Dislocation loops were not readily observed but network of dislocation segments instead. Higher-magnification TEM micrographs were obtained to characterize the size and shape of these cavities. The cavities had multifaceted hexagonal shape with sizes around $5 \mathrm{~nm}$ (Fig. 3a). Some smaller ones were also observed, which was proposed to be the void or the helium bubbles. However, it is really difficult to identify the shape and the inner pressure of these small cavities, so hereafter we will call these defects cavities also. Figure $3 \mathrm{~b}$ shows a hexagonal cavity with its facets aligned with the matrix planes and the corresponding fast Fourier transformation (FFT, inset).

Figure $4 \mathrm{a}, \mathrm{b}$ shows the cavities along the general grain boundary and the twin boundary, respectively. For the general grain boundary, cavities-depleted zone along the grain boundary was readily observed. Besides, TEM micrographs also indicate that dense cavities decorated within the grain boundary plane. However, for the twin boundary, characterized by the selected area electron diffraction (SAED) pattern, cavities were almost homogenously distributed across the twin boundary, suggesting the limited effect of the twin boundary as defect sink compared with the general grain boundary. In the following two sections, the cavity formation along the general grain boundary and the twin boundary was analyzed.

\subsection{Formation and Distribution of the Cavities along General Grain Boundaries}

Figure 5a shows the formation and distribution of the cavities near a grain boundary triple-junction. Cavitiesdepleted zone was clearly observed along the grain boundary, with its width near $20 \mathrm{~nm}$. The variation of density and size of the cavities across the grain boundary can be clearly observed due to the different orientations with respect to the helium ion beam during the irradiation. Figure $5 \mathrm{~b}$ shows two general grain boundaries marked as GB1 and GB2. GB1 and GB2 can be classified as highangle grain boundary and low-angle grain boundary according to the corresponding SAED pattern (insets). Figure $5 \mathrm{c}$ is a zoom-in image of Fig. 5b. Remarkable cavities-depleted zone formed near the GB2 with dense cavities decorated within the GB2 plane. For GB1, we cannot detect the cavities-depleted zone from Fig. 5c, but clearly note the distribution of cavities within the GB1 plane, because it is an inclined grain boundary with respect to the electron beam. The density of the cavities in the GB1 plane was larger than that inside the grain, providing an experimental explanation of the formation of cavities-depleted zone, which will be discussed later. After being titled by a small angle $\left(\sim 10^{\circ}\right)$, the width of GB1 became narrower and cavities-depleted zone was recurred. The 


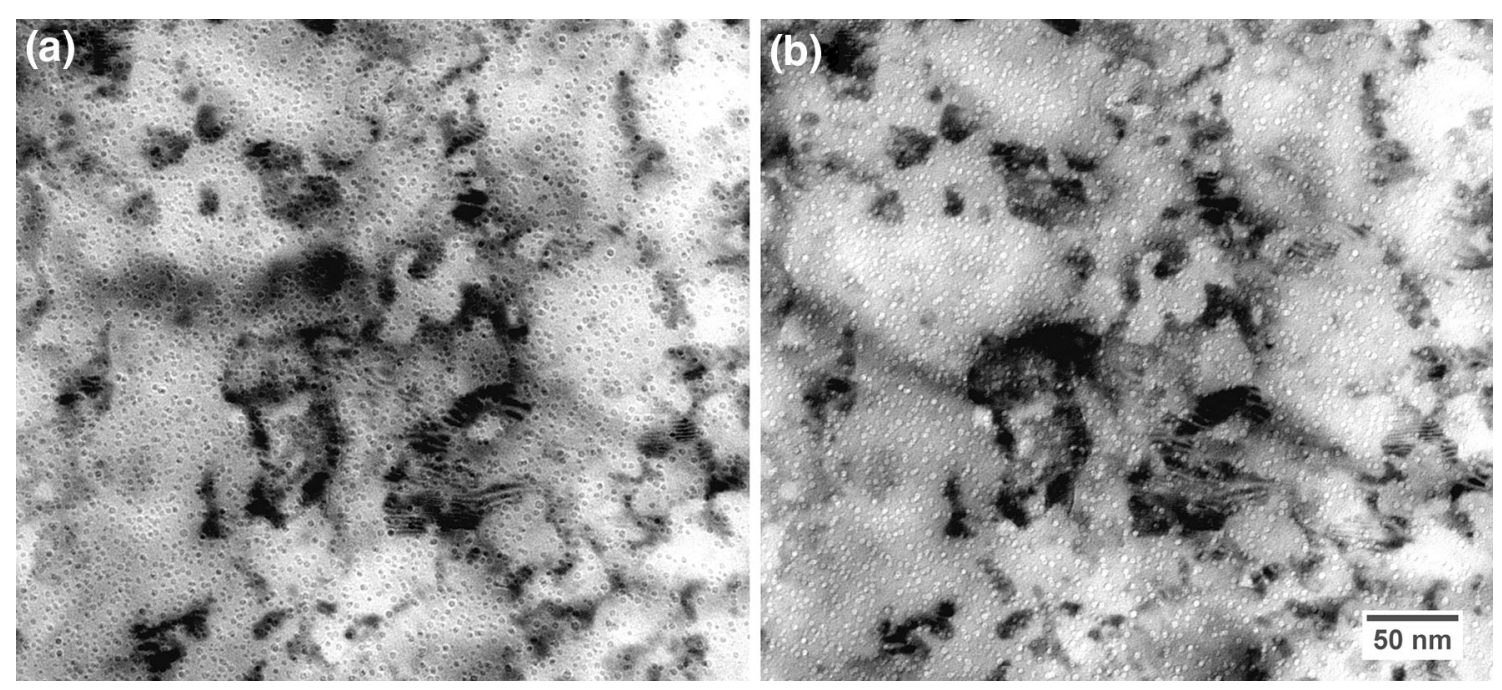

Fig. 2 Cavities formed after helium ion irradiation at room temperature
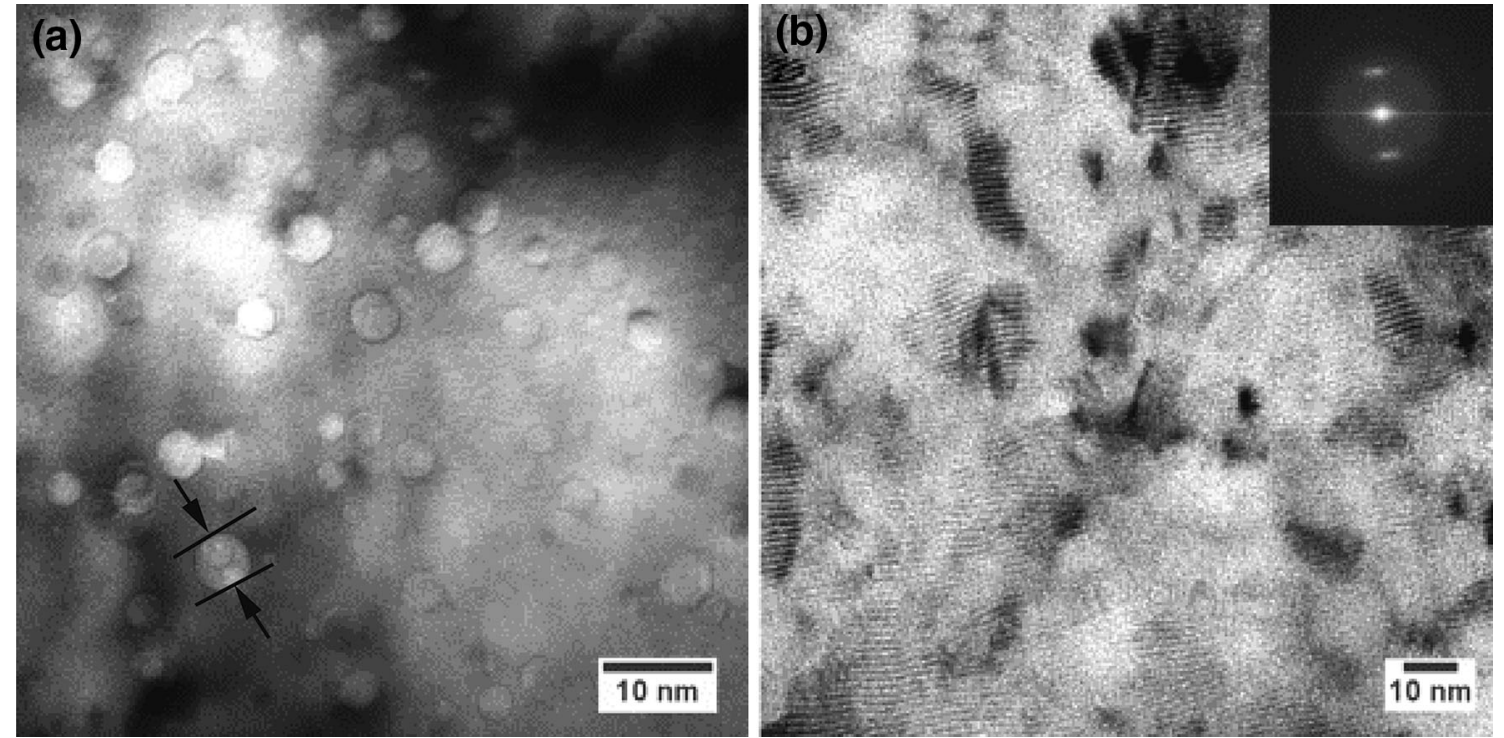

Fig. 3 Cavities formed in the interior of the grain after $30 \mathrm{keV} \mathrm{He}$ ions irradiation at room temperature: a multifaceted shape with size smaller than $5 \mathrm{~nm}$ (measured by the distance shown in the micrograph); b hexagonal cavity with its facets aligned with the matrix. The FFT was superimposed in the inset in $\mathbf{b}$

width of the cavities-depleted zone shown in Fig. $5 \mathrm{c}$ is about $5 \mathrm{~nm}$.

\subsection{Formation and Distribution of the Cavities along Twin Boundaries}

Figure 6a shows the distribution of the cavities along the twin boundary which was characterized by SAED pattern (inset in Fig. 6a) with $\{111\}$ as the twin boundary plane. No cavities-depleted zone was observed along the twin boundary and cavities distributed almost uniformly across the grain boundary, suggesting the limited influence of twin boundary as point defect sink. Figure $6 \mathrm{~b}$ is a magnified micrograph near the twin boundary, showing that no cavities agglomerate and decorate within the twin boundary plane. The number of the cavities in the zone 1 and zone 2 was measured, which will be shown in Sect. 3.4.

\subsection{Comparison between General Grain Boundaries and the Twin Boundaries}

A cavities-depleted zone was observed adjacent to the general grain boundaries. According to Sect. 3.2, the width of the zone is related to the orientation of the grain 

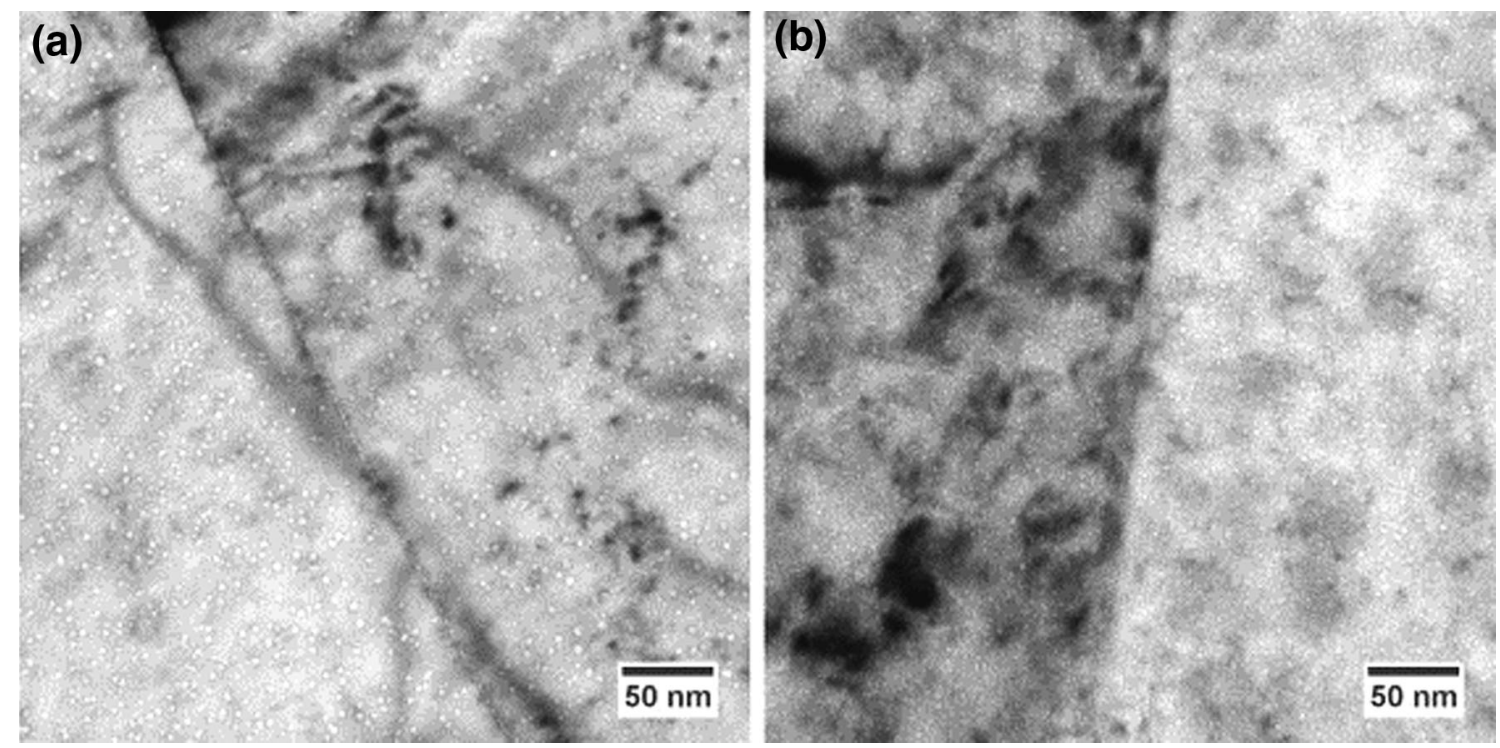

Fig. 4 Cavities near general grain boundary $\mathbf{a}$, twin boundary $\mathbf{b}$ in $\mathrm{Cu}$ after helium irradiation

boundaries. The specimens have to be titled to get the edge-on grain boundary. In order to get the qualitative effect of two different grain boundaries, the number of the cavities in an area of $20 \mathrm{~nm} \times 40 \mathrm{~nm}$ adjacent to the boundaries was counted, as an example shown in Fig. 6 b. Four grain boundaries of both types were randomly selected. The results were plotted and are shown in Fig. 7. For the general grain boundaries, the number of cavities in zone 1 was two times lower than that in zone 2. However, for the twin boundaries, the number of the cavities in both zones was almost in the same level. The width of the cavities-depleted zone also depends on the character of the grain boundaries. The width in GB \#4 was larger so the number of the cavities in zone 1 was fewer than other grain boundaries. For comparison, the number of cavities in two zones when the selected area was $50 \mathrm{~nm} \times 100 \mathrm{~nm}$ is also included in Fig. 7.

\section{Discussion}

\subsection{Effect of Helium on the Cavity Formation}

It is highly mobile for the noble gas helium in most metals $[16,17]$. Small helium cavities can form via helium clustering [18] when no atomic displacement happens. However, when the atomic displacement happens, both the helium atoms and the radiation-induced defects are essential for the formation and growth of helium cavities [19]. Helium atoms will be trapped by vacancies to form $\mathrm{He}-\mathrm{V}$ complex, which will later serve as the nucleation site for helium bubbles or voids. Guo et al. [20] reported the influence of both helium and point defect concentration on the formation of helium-vacancy clusters using KMC simulation method. Previous study has demonstrated that the helium effect accelerates the nucleation of voids in ferritic martensitic material in HT9 [21]. The distribution of helium bubbles in the grains is related to the implantation fluence [22]. In the present work with the high fluence of $1 \times 10^{17}$ ions $/ \mathrm{cm}^{2}$, almost homogenous helium cavities formed in the interior of grain, as shown in Fig. 2.

\subsection{Effect of Grain Boundary on the Cavities- depleted Zone}

It is believed that grain boundaries and interfaces may serve as effective sinks for point defects so they can remove the point defects increasing the radiation tolerance [2]. According to Bai et al. [8], loaded grain boundary will produce interstitial emission removing the bulk vacancies and meanwhile reducing the vacancy diffusion barrier. Therefore, in the region near the grain boundary, vacancies will diffuse to grain boundary or be annihilated by the reemitted interstitials, reducing the concentration of vacancies to form a cavities-depleted zone. The width of those zones depends on the grain boundary characters (compared with Fig. 5a, c). Meanwhile, grain boundary can also serve as sinks for helium atoms [23, 24]. He-V complex diffuses into the grain boundary, and the excess of $\mathrm{He}-\mathrm{V}$ complex agglomerate and cluster to form the cavities within the grain boundary plane. Figure 4a shows a general grain boundary with helium cavities decorating within the grain boundary plane. It should be noted that cavities formed within the grain boundary plane observed here were 

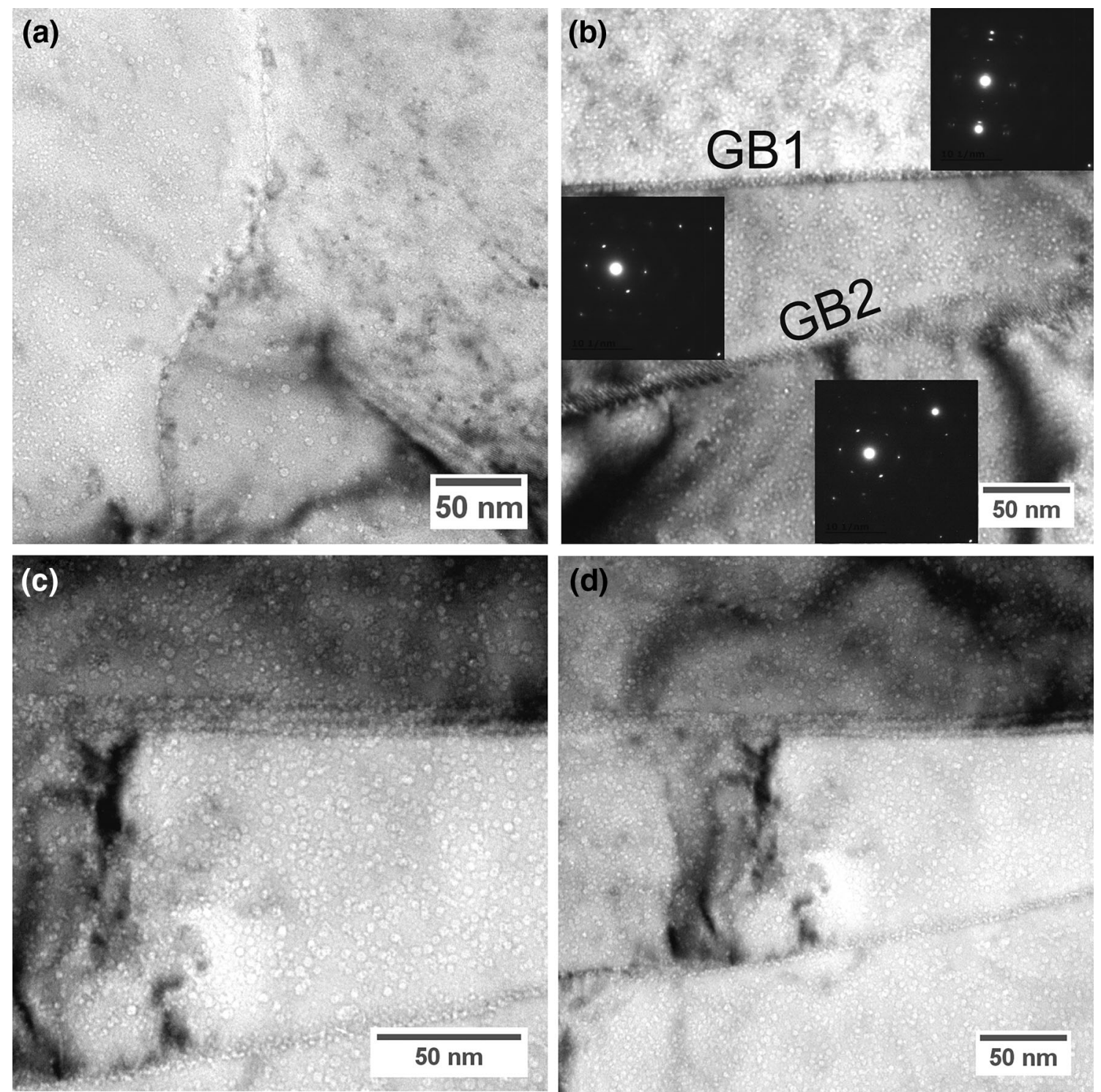

Fig. 5 a Cavity formation near the grain boundary triple-junction; b two general grain boundaries marked as GB1 and GB2, with SAED patterns from the three grains superimposed in the micrograph; c zoom-in image of micrograph $\mathbf{b}$; $\mathbf{d}$ cavity distribution along GB1 after being tilted a small angle $\left(\alpha=8^{\circ}, \beta=7^{\circ}\right)$

detrimental to the material performance, which may induce embrittlement in some alloys $[25,26]$. The high-energy general grain boundaries will produce a higher stress field near the grain boundaries, thus accelerating the interaction between the grain boundaries and the point defects. However, for the twin boundaries, which have the lowest energy, the stress field is weak compared with the general grain boundaries. Thorsen et al. [9] and Han et al. [12] also reported their studies on the bubble formation in heliumion-irradiated copper at elevated temperature. Similar conclusion can be made that general grain boundary had higher efficiency as point defect sink. The main discrepancy with our study is the size of the helium bubbles. In our study, the bubbles had comparable size, while different sizes of bubbles were observed in the interior of grain and near the grain boundary. It should be correlated with the different irradiation temperatures, which constrained the point defects diffusion in our study. From the perspective of the grain boundaries structure, general grain boundaries physically have atom displacement within the grain boundary plane, providing sufficient trapping site for the point defects. Nevertheless, the twin boundaries were twinned grain boundaries and did not have enough trapping sites. The cavities within the twin boundary planes were 

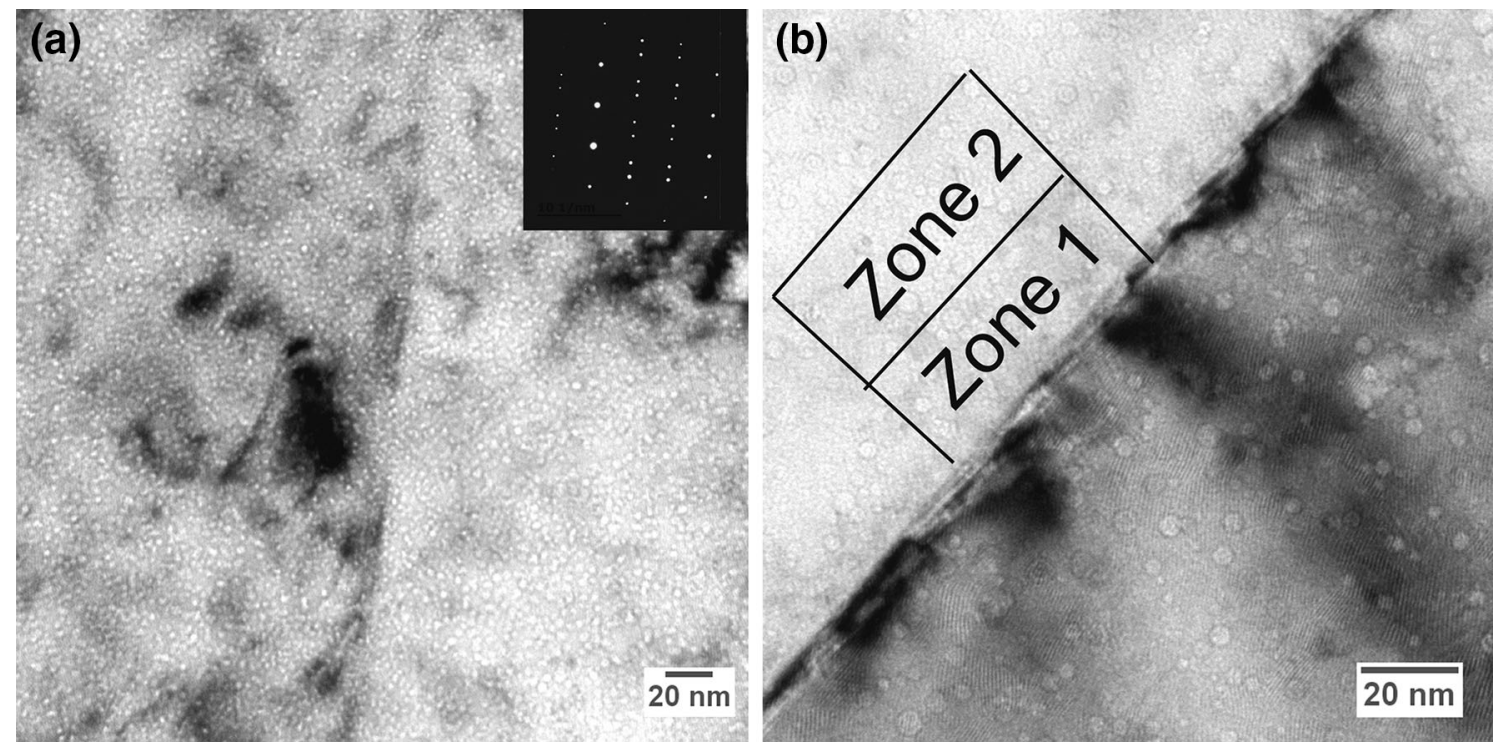

Fig. 6 a Cavities near the twin boundary with its corresponding SAED pattern; b high-magnification micrograph of the cavities near the twin boundary

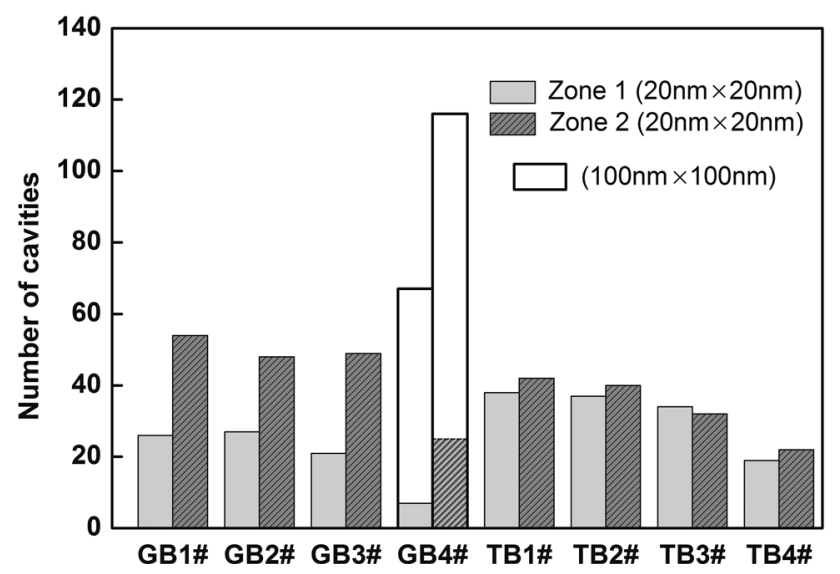

Fig. 7 Statistical data of the cavities number adjacent to two types of the boundaries

seldom observed in this study. Therefore, the twin boundaries showed limited influence as point defect sinks compared with their general grain boundaries counterparts.

\section{Conclusion}

The cavity formation along the general grain boundary and the twin boundary was studied in $\mathrm{Cu}$ followed by helium ion irradiation at room temperature. High density of small cavities formed after radiation. The cavities-depleted zone formed readily near the general grain boundaries. Meanwhile, high density of the cavities was observed within the grain boundary plane. However, for the twin boundaries, no cavities-depleted zone was observed. We concluded that the role of the twin boundaries as point defect sinks was far less than general grain boundaries.

Acknowledgments This work is supported by the National Natural Science Foundation of China (No. 11275023) and the National Magnetic Confinement Fusion Program (No. 2011GB110004).

\section{References}

[1] S.J. Zinkle, Fusion Eng. Des. 74, 31 (2005)

[2] L. Liu, Z. Tang, W. Xiao, Z. Wang, Mater. Lett. 109, 221 (2013)

[3] A. Alsabbagh, A. Sarkar, B. Miller, J. Burns, L. Squires, D. Porter, J.I. Cole, K.L. Murty, Mater. Sci. Eng. A 615, 128 (2014)

[4] T.D. Shen, S. Feng, M. Tang, J.A. Valdez, Y. Wang, K.E. Sickafus, Appl. Phys. Lett. 90, 263115 (2007)

[5] H. Tsuchida, T. Iwai, M. Awano, N. Oshima, R. Suzuki, K. Yasuda, C. Batchuluun, A. Itoh, J. Nucl. Mater. 442, S856 (2013)

[6] Y. Chimi, A. Iwase, N. Ishikawa, M. Kobiyama, T. Inami, S. Okuda, J. Nucl. Mater. 297, 355 (2001)

[7] N. Nita, R. Schaeublin, M. Victoria, J. Nucl. Mater. 329-333, 953 (2004)

[8] X.M. Bai, A.F. Voter, R.G. Hoagland, M. Nastasi, B.P. Uberuaga, Science 327, 1631 (2010)

[9] P.A. Thorsen, J.B. Bilde-Sørensen, B.N. Singh, Scr. Mater. 51, 557 (2004)

[10] N. Sakaguchi, M. Endo, S. Watanabe, H. Kinoshita, S. Yamashita, H. Kokawa, J. Nucl. Mater. 434, 65 (2013)

[11] M. Tomozawa, Y. Miyahara, K. Kako, Mater. Sci. Eng. A 578, 167 (2013)

[12] W.Z. Han, M.J. Demkowicz, E.G. Fu, Y.Q. Wang, A. Misra, Acta Mater. 60, 6341 (2012)

[13] S.I. Porollo, A.M. Dvoriashin, Y.V. Konobeev, F.A. Garner, J. Nucl. Mater. 442, S809 (2013)

[14] D. Yun, M.A. Kirk, P.M. Baldo, J. Rest, A.M. Yacout, Z.Z. Insepov, J. Nucl. Mater. 437, 240 (2013)

[15] B.V. Cockeram, R.W. Smith, N. Hashimoto, L.L. Snead, J. Nucl. Mater. 418, 121 (2011) 
[16] X. Shu, P. Tao, X. Li, Y. Yu, Nucl. Instrum. Methods Phys. Res. Sect. B 303, 84 (2013)

[17] D. Stewart, Y. Osetskiy, R. Stoller, J. Nucl. Mater. 417, 1110 (2011)

[18] X. Gai, T. Lazauskas, R. Smith, S.D. Kenny, J. Nucl. Mater. 462, 382 (2014)

[19] V. Raineri, S. Coffa, M. Saggio, F. Frisina, E. Rimini, Nucl. Instrum. Methods Phys. Res. Sect. B 147, 292 (1999)

[20] X. Guo, X. Zhang, J. Xue, W. Li, Nucl. Instrum. Methods Phys. Res. Sect. B 307, 77 (2013)

[21] E. Getto, Z. Jiao, A.M. Monterrosa, K. Sun, G.S. Was, J. Nucl. Mater. 462, 458 (2015)
[22] B.S. Li, Y.Y. Du, Z.G. Wang, K.F. Wei, H.P. Zhang, C.F. Yao, H.L. Chang, J.R. Sun, M.H. Cui, Y.B. Sheng, L.L. Pang, Y.B. Zhu, X. Gao, P. Luo, H.P. Zhu, J. Wang, D. Wang, Vacuum 113, 75 (2015)

[23] F. Sefta, K.D. Hammond, N. Juslin, B.D. Wirth, Nucl. Fusion 53, 073015 (2013)

[24] O. El-Atwani, K. Hattar, J.A. Hinks, G. Greaves, S.S. Harilal, A. Hassanein, J. Nucl. Mater. 458, 216 (2015)

[25] S.I. Porollo, A.N. Vorobjev, Y.V. Konobeev, A.M. Dvoriashin, V.M. Krigan, N.I. Budylkin, E.G. Mironova, F.A. Garner, J. Nucl. Mater. 258-263, 1613 (1998)

[26] D.J. Edwards, F.A. Garner, S.M. Bruemmer, P. Efsing, J. Nucl. Mater. 384, 249 (2009) 